\title{
Researches regarding introducing temperature as a factor in cutting tool wear monitoring
}

\author{
Marinela Inţă ${ }^{*}$ and Achim Muntean \\ Lucian Blaga University of Sibiu, Industrial Engineering and Management Department, 550025, B- \\ dul Victoriei, no.10, Sibiu, Romania
}

\begin{abstract}
Monitoring the tool state during the cutting process becomes very important since the introduction of CAM in manufacturing process. The tool life based only on economic data is not enough especially in the case precision cutting due to the scattering of tool wear which depends on much more mechanical and physical properties of the workpiece and tool materials. Attempts to assess the state of wear of the tool by direct measurement of the wear indicators have been unsuccessful due to difficult access in the cutting zone and measurement imprecision. We have so wear patterns appreciation by measuring indirect indicators of wear, the main models are based on measuring vibration, acoustic emission or cutting forces and power consumption separately or together. These models have the disadvantage that should be followed at least two indicators simultaneously for a minimum precision of the results. Considering the wear models developed over time it can be seen that there is a clear relationship between wear and stabilized temperature. The paper aims to study the introduction temperature as indirect indicator of tool state in addition to the existing indicators, this leads to increased reliability of results of monitoring of wear.
\end{abstract}

\section{Introduction}

During the machining process, in the contact area of the tool and the workpiece, and in the shear aria thermal phenomena occur because almost all the mechanical work is transformed into heat and only a small part is stored in the form of potential energy through the remaining tensions in the metallic crystal network. By distributing the heat to the chip, the tool, the piece and the environment, their temperature increases in proportion to the percentage of total heat absorbed. Among the listed items, the increase in tool temperature leads to lower cutting properties and increased wear.

The cutting tool temperature is one of the most important elements from a practical point of view because it leads to deterioration of the cutting properties of the cutting edge, increasing the wear and decreasing tool life, introducing residual stresses into the machined surface with important economic effects in the cutting process. Despite the high level of

${ }^{*}$ Corresponding author: marinela.inta@ulbsibiu.ro 
existing technology for each type of processing, there is still a factor that prevents the safety and complete automation of processes. This factor is tool wear.

We consider that the wear reduction by the optimization of the cutting regime has been adequately studied and the results have reached considerable values, but for monitoring the tool condition in the automated machine processing it is more important to study the admissible wear threshold if this optimization. For this reason, over the years several ways of approaching the tool's state and, implicitly, its wear have been developed by finding indirect wear indicators. However, often the methods used do not meet all the requirements. One of the indirect indicators, the temperature and the heat flux released in both the cutting tool and the cutting edge has not been extensively researched because of the complexity of the tool geometry and the direct access to the interface plate-tool shaft.

Numerous attempts have been made to address the problem through various analytical and experimental methods. Although considerable research efforts have been made on the thermal problem of metal cutting.

In the paper [1], the authors present a new analytical method regarding the temperature of the cutting process and the heat generated in the tool-piece-chip when milling special materials. They use a semiartificial thermocouple (single-threaded) to measure the temperature. Komanduri and Hou in the paper [2] analyzed in detail the temperature using the method developed by Jaeger, calculating only the temperatures of the chips and the workpiece caused by the heat source in the shear plane. G. List et al. [3] investigated the interface temperature and showed dependence on the wear mechanism at high speed cutting. They calculated the shear stress and the heat shared on the rake face by Shaw method and then by FEM analyzed the temperature distribution. In paper [4], research on heat generation and dissipation of heat in the orthogonal process is critically analyzed.

Papers [5-6] have measured and compared the temperature during turning at different speeds and feeds using covered and uncovered tungsten carbide plates and the method of the K-type thermocouple. They concluded that tool temperature reduction improves both lifetime and roughness of the work surface.

Ay and Yang [7] used a K-type thermocouple technique to analyze the temperature variations of the carbide plates for cutting various materials such as copper, cast iron, aluminum 6061 and AISI 1045 steel. They observed that the oscillations at temperatures in locations near the cutting edge were more rigorous for ductile materials and less hardmachining materials. In study [8], the tool temperature was estimated by simultaneous measurement, using both a K-type thermocouple and an infrared (IR) pyrometer.

In the present paper we propose the experimental model based on measuring the temperature of the junction tool-chip and tool-piece, by the method of natural thermocouple, considering it to be the most appropriate because it measures the average junction temperature without introducing changes in the tool or the piece, the cutting process is not influenced constructively by the elements of the method.

For verification and comparison, the infrared camera is simultaneously used using the orthogonal cutting and the cutting area with the simultaneous view of tool edge and piece. For the particularization of the theoretical models obtained by the FEM method it is necessary to carry out experimental researches under the same conditions in which simulation and modeling of the cutting process was done in the case of the turning operation.

\section{Experimental researches}

Experimental tests for the temperature measurement of the cutting process were performed for the turning operation on X6CrNiMoB17-12-2 (corresponding to AISI 316h machining) material $50 \mathrm{~mm}$ in diameter using a CNMM 150404 carbide plate with titanium nitride 
coating on which the influence of cutting speed, cutting depth and feed was analyzed. Frontal turning was used because it simulates orthogonal cutting.

In the tests two methods were used: the natural thermocouple and a thermography camera (IR), in simultaneous recording. The measurement system is shown in Figure 1.
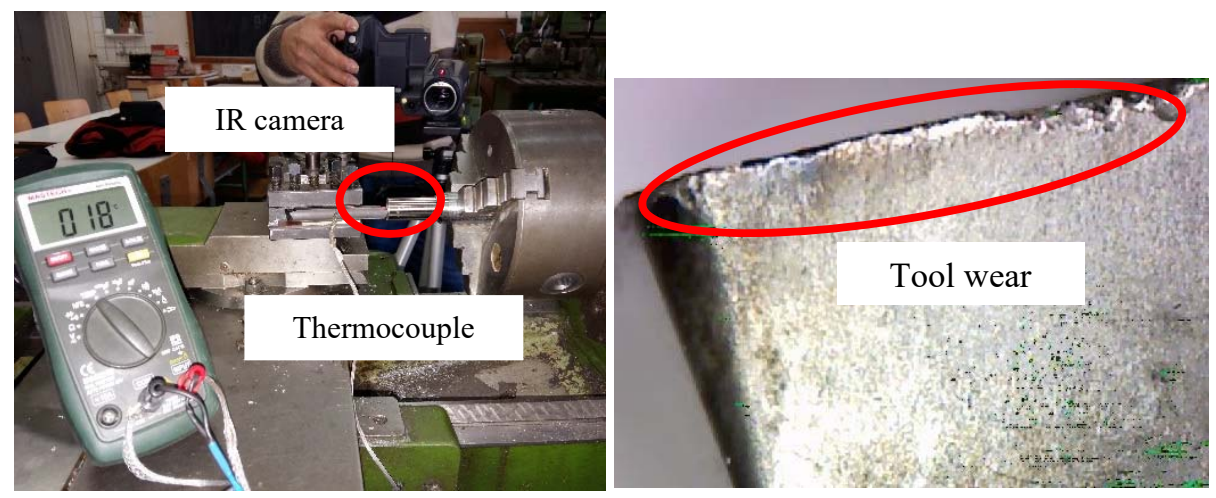

Fig. 1. Measurement of temperature and tool wear.

In determining factor levels, a factorial experiment $2^{3}$ was selected which comprises three factors (feed, cutting speed and cutting depth) at two levels of variation.

Table 1. Experimental conditions.

\begin{tabular}{|l|c|c|c|}
\hline Parameter & Coded & \multicolumn{2}{|c|}{ Values } \\
\hline Real & $\mathrm{X} 1$ & 0.075 & 0.15 \\
\hline Feed, $\mathbf{f}[\mathbf{m m} / \mathbf{r o t}]$ & $\mathrm{X} 2$ & 0.5 & 0.1 \\
\hline Depth of cut, $\mathbf{a}_{\mathbf{p}}[\mathbf{m m}]$ & $\mathrm{X} 3$ & 55 & 80 \\
\hline Cutting speed, $\mathbf{v}_{\mathbf{c}}[\mathbf{m} / \mathbf{m i n}]$ & \multicolumn{3}{|}{} \\
\hline
\end{tabular}

\subsection{Temperature measurement with natural thermocouple}

The use of the natural thermocouple involves the insulation of the workpiece and the tool against the machine tool structure (Figure 2). Hot junction is precisely the contact between the tip of the cutting tool and the workpiece. Measuring the voltage generated by this junction (transformed in temperature by calibration diagram) take by the cold contacts, from material and the body of the tool, we have a measure that characterizes the actual temperature that occurs at the contact point. The IR camera is placed on lateral size recording in the same time (Bothroyd method).

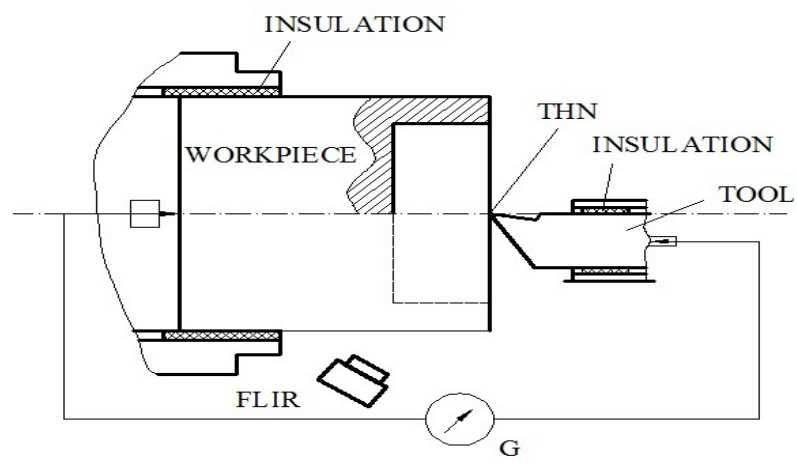

Fig. 2. Measurement of the temperature in the cutting area by natural thermocouple method and thermography. 


\subsection{Temperature measurement with infrared camera}

A FLIR T440 infrared camera (IR) is used to observe the temperature of the cutting process by viewing both the piece and the cutting tool in a simulation of an orthogonal cutting. The infrared camera records images at $30 \mathrm{~Hz}(30$ frames / sec). The camera has been set to measure the process temperature within a range of $20-800^{\circ} \mathrm{C}$.

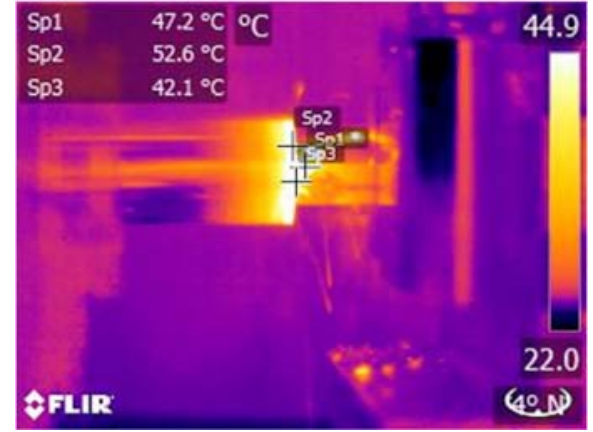

a) during cutting process

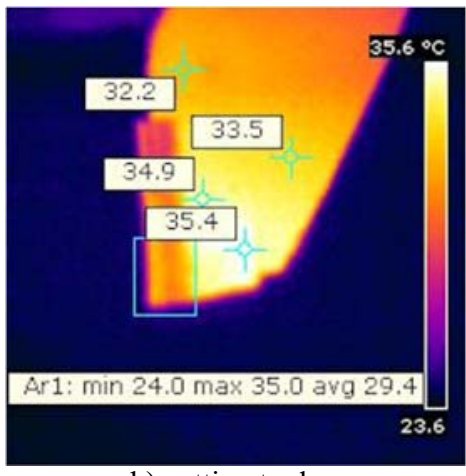

b) cutting tool

Fig. 3. Temperature measuring using IR camera.

The recording was performed simultaneously with the temperature measurement using the natural thermocouple. The resulting images were then processed with FLIR Tools software. In Figure 3 the thermographic images, with the analysis of a sequence. Also the temperature measured with IR camera is higher due to the fact that camera points a bigger area than the contact tool-workpiece.

\subsection{Analysis of experimental results}

Using the two methods of measuring the temperature at the turning operation, experimental results were obtained from the following table.

Table 2. Experimental results.

\begin{tabular}{|c|c|c|c|c|c|}
\hline No & $\mathrm{a}_{\mathrm{p}}[\mathrm{mm}]$ & $\mathrm{f}[\mathrm{mm} / \mathrm{rot}]$ & $\mathrm{V}_{\mathrm{c}}[\mathrm{m} / \mathrm{min}]$ & $\begin{array}{c}\text { Temperature }\left[{ }^{0} \mathrm{C}\right] \\
\text { Thermocouple }\end{array}$ & $\begin{array}{c}\text { Temperature }\left[{ }^{\circ} \mathrm{C}\right] \\
\text { FLIR }\end{array}$ \\
\hline 1. & \multirow{4}{*}{0.5} & \multirow{2}{*}{0.075} & 55 & 38 & 42.1 \\
\hline 2. & & & 80 & 52 & 52.6 \\
\hline 3. & & \multirow{2}{*}{0.15} & 55 & 40 & 47.2 \\
\hline 4. & & & 80 & 61 & 68.2 \\
\hline 5. & \multirow{4}{*}{1} & \multirow{2}{*}{0.075} & 55 & 73 & 89.6 \\
\hline 6. & & & 80 & 85 & 109.1 \\
\hline 7. & & \multirow{2}{*}{0.15} & 55 & 96 & 113.6 \\
\hline 8. & & & 80 & 108 & 132.9 \\
\hline
\end{tabular}

As it can be seen the Table 2, the temperature increase is higher with the increase of cutting speed than feed increase, this because the feed has a great influence on the chiptool contact area on the rake face.

\section{Finite Element Simulation of Cutting tools temperature}

The finite element method was applied during time from the analysis of the perfect elastic media to the analysis of the elastoplastic media. This evolution allowed the use of the method in the shaping of the cutting process. Thus, the researchers have proposed wear patterns that report wear rate to temperature, [9]. 
In the present paper, in order to see the temperature distribution and the wear of the cutting tool, a series of simulations were made in the Deform 3D Machining software under the same conditions in which the experimental tests were carried out, mainly by looking at the temperature in the tool cutting. Thus, in the simulation of the turning process, a WC tungsten carbide plate, coated with a layer of 5 microns of titanium carbonate (TiCN) was used, and as a piece of material, AISI $316 \mathrm{~h}$ machining having a working diameter of $\mathrm{D}=50 \mathrm{~mm}$. As a model for calculating the tool wear, Usui's model, [eq.1, 10] is used where the coefficients $a$ and $b$ depend on the material used and have the values: $a=0.0000002$ and $b$ $=650.5$.

$$
w=\int a \cdot p \cdot v \cdot e^{-b / T} \cdot d t
$$

Where: $\quad w-$ tool wear

$\mathrm{v}$ - sliding velocity, [m/min],

$\mathrm{T}$ - temperature $\left[{ }^{0} \mathrm{C}\right]$

$\mathrm{p}$ - interface pressure

dt - time increment.
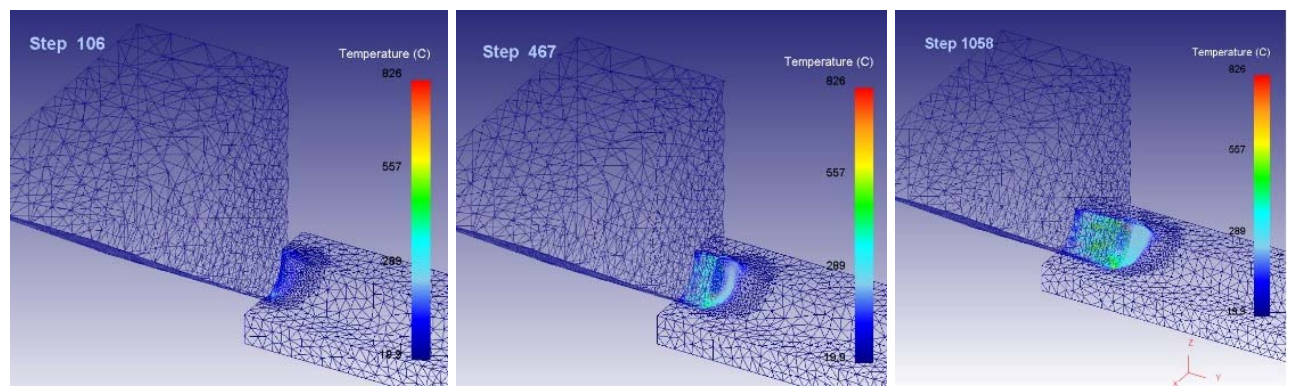

Fig. 4. Distribution of temperature in the contact zone.
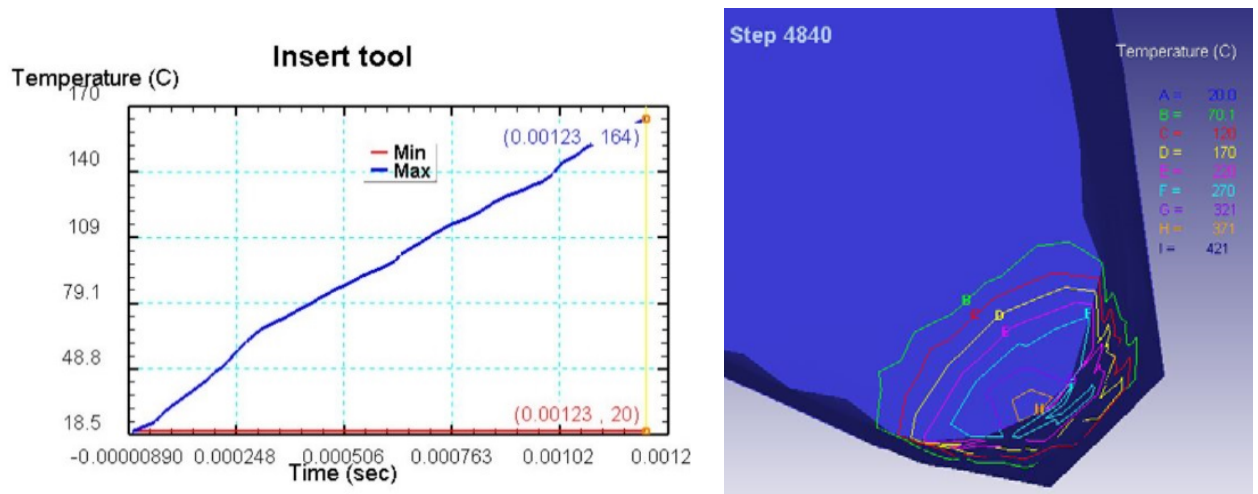

Fig. 5. The temperature in the tool nodes under stationary conditions (no. 8 from Table 2).

As a result of the simulations performed (Figure 4), it is observed that the temperature of the nodes at the rake face of the tool in the stationary thermal regime is obtained by analysis of the heat transfer.

After running the program, it can be noticed that the crater of the wear on the rake face looks like with representation of the isotherms, that showing that the maximum depth of wear crater corresponds with maximum of temperature. The isotherms of temperature are presented in Figure 5. 


\section{Conclusions}

It has been found that the application of simultaneous measurements (both by the IR camera and the natural thermocouple) in the processing is unique and there are very few related works found in the literature. For the validation of the theoretical model obtained through FEM, the experimental tests were also performed for the turning operation for which the modeling was performed under the same cutting conditions and on the same workpiece. During the experiment the duration of the cutting was only 10 seconds similar with the one used in simulation, though the temperature does not reach the stationary condition in the experiment. Graphical representation of the temperature values, experimentally measured by the two methods (Figure 6) it can be observed that measured temperature with IR camera is higher than that with the natural thermocouple, the tendency being the same and the differences being relatively small. Temperature values obtained experimentally are coherent to those obtained by simulating with Deform 3D software. Thus, this method can be improved from the point of view of precision and used to measure on-line temperature in metal cutting. While some cases indicate a relationship between the tool condition and the radiant temperature profile, there is not enough evidence at this time to draw any conclusions.

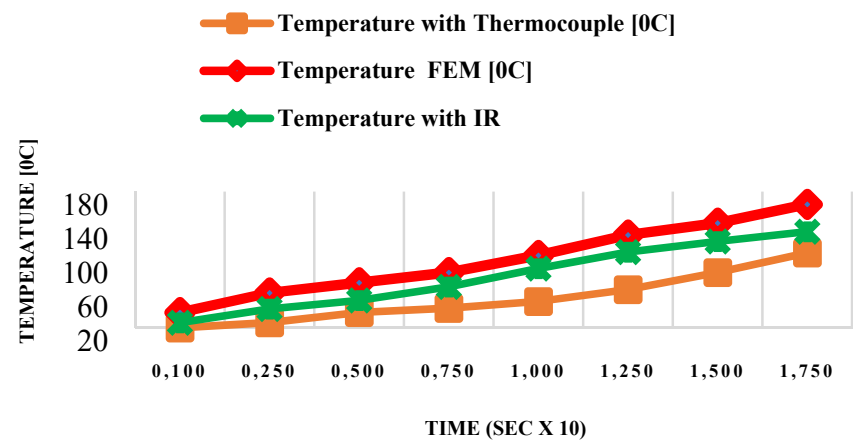

Fig. 6. Temperature variation for all the cutting conditions.

\section{References}

1. C. Di, Z. Dinghua, W. Baohai, L. Ming. Procedia CIRP 58, 49 - 54 (2017)

2. R. Komanduri, ZB. Hou.. Int J Mech Sci 42, 1715-1752 (2000)

3. G. List, G. Sutter, A. Bouthiche. Int J Mach Tool Manu 54-55:1-9 (2012)

4. N. Abukhshim, P. Mativenga. M. Aslam Sheikh, International Journal of Machine Tools and Manufacture 46 (7-8), 782-800 (2006)

5. W. Baohai C. Di He Xiaodong Zhang Dinghua Tang Kai. Chinese J Aeronaut 29, 17881794 (2016)

6. D. Sushil Ghodam. IJRET, 3(4), 831-835 (2014)

7. H. Ay. W.J. Yang. Int. J. Heat Mass Trans. 41, 613-623 (1998)

8. Abdil Kus, Yahya Isik, M. C. Cakir, S. Coşkun, and K., Sensors (Basel), 15(1), 12741291 (2015)

9. E. Uhlmanna, R. Gerstenbergera, J. Kuhnertb, Procedia CIRP. 8, 391-396 (2013)

10. M. Inţă, A. Muntean, C. Petrianu, Proceedings of the $6^{\text {th }}$ Balkan Region Conference on engineering and Business Educationn (DE GRUYTER OPEN, 107-110 2012) 